Mehr, S. A., Singh, M., Knox, D., Ketter, D., Pickens-Jones, D., Atwood, S., ..., \& Glowacki, L. (2019). Universality and diversity in human song. Science, 366(970), 957-970. https://doi.org/10.31234/osf.io/emq8r.

Oesch, N. (2019). Music and language in social interaction: Synchrony, antiphony, and functional origins. Frontiers in Psychology, 10, article 1514, 1-13. https://doi.org/10. 3389/fpsyg.2019.01514.

Oesch, N. (2020). Evolutionary musicology. In T. K. Shackelford \& V. A. Weekes-Shackelford (Eds.), Encyclopedia of evolutionary psychological science (pp. 1-6). Springer International Publishing. https://doi.org/10.1007/978-3-319-16999-6_2845-1.

Popescu, T., Neuser, M. P., Neuwirth, M., Bravo, F., Mende, W., Boneh, O., Moss, F. C., \& Rohrmeier, M. (2019). The pleasantness of sensory dissonance is mediated by musical style and expertise. Scientific Reports, 9(1), 1070-1080. https://doi.org/10.1038/s41598018-35873-8.

Ridley, M. (2003). Evolution (3rd ed.). Wiley.

Schoenberg, A. (2010). Style and idea: Selected writings. University of California Press.

Sugimoto, T., Kobayashi, H., Nobuyoshi, N., Kiriyama, Y., Takeshita, H., Nakamura, T., \& Hashiya, K. (2009). Preference for consonant music over dissonant music by an infant chimpanzee. Primates, 51(1), 7. https://doi.org/10.1007/s10329-009-0160-3.

Trainor, L. J., Tsang, C. D., \& Cheung, V. H. W. (2002). Preference for sensory consonance in 2- and 4-month-old infants. Music Perception: An Interdisciplinary Journal, 20(2), 187-194. https://doi.org/10.1525/mp.2002.20.2.187.

Watanabe, S., Uozumi, M., \& Tanaka, N. (2005). Discrimination of consonance and dissonance in Java sparrows. Behavioural Processes, 70(2), 203-208. https://doi.org/10. 1016/j.beproc.2005.06.001.

Whittingham, L. A., Kirkconnell, A., \& Ratcliffe, L. M. (1997). The context and function of duet and solo songs in the red-shouldered blackbird. The Wilson Bulletin, 109, 279-289.

\section{Isochrony, vocal learning, and the acquisition of rhythm and melody}

\section{Andrea Ravignani $\odot$}

Comparative Bioacoustics Group, Max Planck Institute for Psycholinguistics, Nijmegen, 6525 XD, The Netherlands.

andrea.ravignani@mpi.nl; https://www.mpi.nl/people/ravignani-andrea

\section{doi:10.1017/S0140525X20001478, e88}

\begin{abstract}
A cross-species perspective can extend and provide testable predictions for Savage et al.'s framework. Rhythm and melody, I argue, could bootstrap each other in the evolution of musicality. Isochrony may function as a temporal grid to support rehearsing and learning modulated, pitched vocalizations. Once this melodic plasticity is acquired, focus can shift back to refining rhythm processing and beat induction.
\end{abstract}

Musicality consists of the (neuro)biological underpinnings to perceive and produce music. Research in the evolution of musicality needs cross-species evidence. As a parallel, to understand the evolution of bat wings, one asks why all other mammals lack wings and why other flying animals have evolved them. Similarly, our species only constitutes one datapoint to construct evolutionary hypotheses on musicality. Comparisons with other species are necessary to avoid post-hoc explanations of evolutionary traits.

Four concepts discussed in Savage et al. are key for understanding musicality, both in humans and other animals (Fig. 1). Isochrony describes metronomic temporal regularity, similar to the ticking of a clock (Merker, Madison, \& Eckerdal, 2009; Ravignani \& Madison, 2017). Synchrony is the perfect co-occurrence in time of two series of events, with no strong teleological or mechanistic focus (Kotz, Ravignani, \& Fitch, 2018;
Ravignani, 2017). Vocal learning is the ability to learn and modify non-innate vocalizations, including melodies (Lattenkamp \& Vernes, 2018). Beat induction denotes a top-down capacity to induce a regular pulse from music and move in synchrony to it (Grahn \& Brett, 2007; Honing, 2012).

Do other animals have these capacities supporting musicality? Isochrony appears in many species' communication (e.g., from lobster rattles to sea lion barks: Patek \& Caldwell, 2006; Schusterman, 1977), autonomously-regulated behavior or (neuro)physiology. Synchrony is widespread but scattered across taxonomic groups (Ravignani, Bowling, \& Fitch, 2014; Wilson \& Cook, 2016). Vocal learning is rare but potentially arose multiple times in evolution because of different pressures across species (Garcia \& Ravignani, 2020; Martins \& Boeckx, 2020; Nowicki \& Searcy, 2014). Beat induction has only been found in a few animals, as acknowledged by Savage and colleagues (Kotz et al., 2018; cf. Mehr et al., claiming its presence in many species).

Savage and colleagues briefly characterize these four abilities; this invites discussion of cross-species implications and predictions as to how they evolved to support musicality. I add a fifth, still largely unexplored capacity: vocal rhythms, which consist of producing, perceiving, learning, or imitating signals with accuracy in the temporal - as opposed to the spectral - domain. Although this capacity to precisely time one's vocalizations is related to its spectral counterpart, vocal rhythms also have their own mechanistic and communicative value (Wirthlin et al., 2019). I argue that, across species, these five capacities are linked, mapping them to Savage et al.'s framework.

The core of Savage et al.'s idea of melodic and rhythmic musicality features vocal learning and beat induction. These are also at the core of an influential hypothesis in evolutionary neuroscience (Patel, 2006), predicting in some cases their joint co-occurrence across species. However, a few outlier species point to a mismatch between the current data and the hypothesis' predictions (Cook, Rouse, Wilson, \& Reichmuth, 2013), requiring an updated theoretical framework.

Within Savage et al.'s framework, I argue that rhythm and melody may have bootstrapped each other in humans and other species gradually, especially in social interactions, such as chorusing, turn-taking, and so forth (Christophe, Millotte, Bernal, \& Lidz, 2008; Hannon \& Johnson, 2005; Höhle, 2009; Ravignani et al., 2014). An isochronous sequence, such as the repetitive bark of a sea lion, provides a temporal grid of predictable sound events. Both the producer of an isochronous rhythm and its conspecifics can rely on this periodicity to learn and experiment in the spectral, hence melodic, domain during vocal learning: vocal emissions could be anchored to the onsets of the isochronous sequence (Merker et al., 2009). Hence, rhythmic isochrony may function as temporal grid to rehearse learnt vocalizations (and possibly orient attention; Bolger, Coull, \& Schön, 2014; Cason, Astésano, \& Schön, 2015; Jones, 2010; Norton, 2019). In turn, learnt, consolidated vocalizations may serve as a "spectral anchor" to segment conspecifics' temporal sequences (Hyland Bruno, 2017; Lipkind et al., 2013), also generating vocal rhythms. Therefore, melodic templates acquired via vocal learning can afford increased attentional or cognitive resources spent on the rhythmic domain, including temporal segmentation and regularization. This provides a bootstrapping mechanism for Savage et al.'s co-evolutionary dynamics to work, and a testbench for some signaling hypotheses in Mehr and colleagues.

This hypothesis generates several testable predictions. First, by testing species along the vocal learning continuum (Martins \& Boeckx, 2020), and extending this continuum to beat induction, 


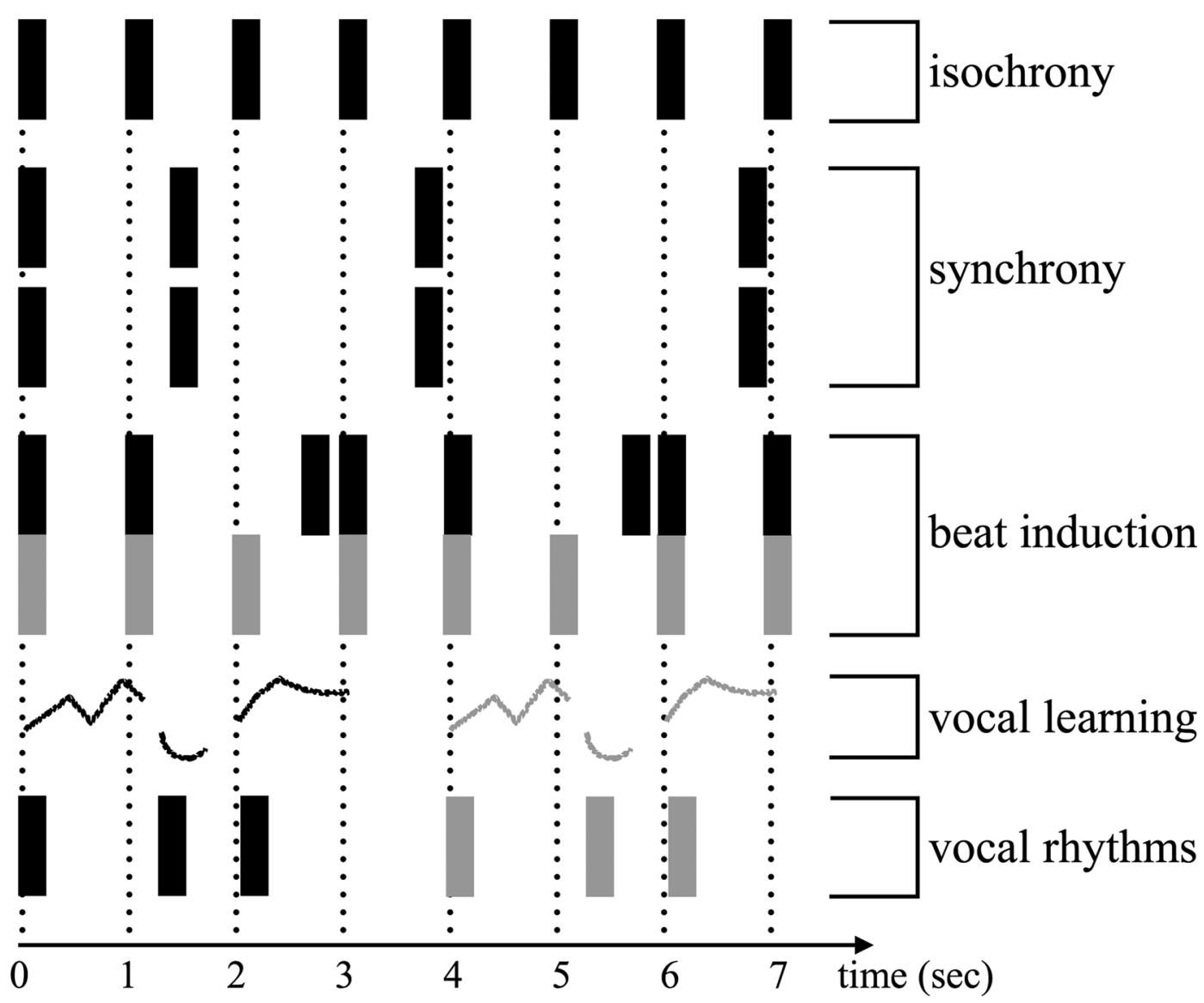

Figure 1. (Ravignani) Conceptualization of the four abilities partly explored in the target articles plus a fifth one, vocal rhythms, which deserves entering the discussion. Isochrony, when present in acoustic or motoric behaviors, may provide a clear, extremely predictable temporal grid, similar to squared notebooks guiding children who learn how to write. An isochronous pattern is, per se, neither musical nor demanding to produce or perceive. Isochrony has low entropy, definitely lower than expected for "musical" patterns (Milne \& Herff, 2020; Ravignani \& Madison, 2017). Production of isochrony can result from a motoric behavior entraining to a neural oscillator. Perception of isochrony requires, at least, comparing pairs of temporal intervals, an ability found in several species (e.g., Church \& Lacourse, 1998; Heinrich, Ravignani, \& Hanke, 2020; Ng, Garcia, Dyer, \& Stuart-Fox, 2020). Although isochrony is characterized by equal timing in a series of events, synchrony requires pairwise coincidence of events from two series, neither of which needs to be isochronous (Ravignani, 2017). Given an acoustic sequence (black), beat induction consists of inferring an isochronous pulse (gray), which need not physically exist in the sequence (Honing, 2012; Kotz et al., 2018). Synchronization differs from beat induction in being independent from isochrony, relatively inflexible, achievable for a narrow range of tempi and unimodal (Patel, Iversen, Bregman, \& Schulz, 2009). Vocal learning - here with emphasis in its spectral domain - includes, among other things, the capacity to copy (gray) a vocal signal (black) (Lattenkamp \& Vernes, 2018; Wirthlin et al., 2019). A vocal rhythm (black) is a temporal pattern of events, which conveys most information in the temporal domain (Ravignani et al., 2019) and could also be learnt or imitated (gray).

species with a stronger sense of beat should be found among those with more developed vocal learning capacities. Chickens, great apes, parrots, and humans are examples of species predicted to show, in this order, increasing abilities in both domains. Second, isochrony should go hand in hand with synchrony but not with beat induction, so that species with developed isochrony should also synchronize. Third, empirical evidence for the rhythm-melody scaffolding process (Cason et al., 2012; Emmendorfer, Correia, Jansma, Kotz, \& Bonte, 2020) could be obtained from large-scale developmental datasets, which should feature both humans and nonhuman animals, and contain data from as many capacities as possible from Figure 1. As ontogeny sometimes recapitulates phylogeny (e.g., Heldstab, Isler, Schuppli, \& van Schaik, 2020), one would test whether the same stepwise processes hypothesized above appear in the first years of human life (Höhle, 2009). Fourth, a partial neural dissociation between rhythm and melody may occur early in life and become less severe over development; the dynamics of this dissociation could be tested via longitudinal neuroimaging studies (Bengtsson \& Ullén, 2006; Salami, Wåhlin, Kaboodvand,
Lundquist, \& Nyberg, 2016). Fifth, within Savage et al.'s framework, physiological evidence for the rhythm-melody gradual interplay could come from measurements or manipulations of the dopaminergic reward system and the endogenous opioid system, testing whether they provide complementary, alternating effects. Finally, most of these putative links can be, following Savage et al., modulated by species-specific social factors, such us group density and social networks. Similarly, their value as honest signals can be tested to provide empirical support for Mehr et al. using, among others, methods from cultural evolution research (e.g., Lumaca et al., commentary on the target article by Mehr et al.; Miton, Vesper, Wolf, Knoblich, \& Sperber, 2020).

To conclude, the frameworks proposed in both target articles can benefit from a finer dissection of core abilities for musicality (Fig. 1 and Honing, commentary on the target article by Savage et al.). These must then be tested across species to infer plausible evolutionary scenarios.

Acknowledgments. I am grateful to Henkjan Honing, Koen de Reus, Laura Verga, Massimo Lumaca, and Sonja Kotz for helpful discussion and feedback. 
Financial support. Andrea Ravignani is supported by the Max Planck Society via an Independent Research Group Leader position.

Conflict of interest. None.

\section{References}

Bengtsson, S. L., \& Ullén, F. (2006). Dissociation between melodic and rhythmic processing during piano performance from musical scores. NeuroImage, 30(1), 272-284.

Bolger, D., Coull, J. T., \& Schön, D. (2014). Metrical rhythm implicitly orients attention in time as indexed by improved target detection and left inferior parietal activation. Journal of Cognitive Neuroscience, 26(3), 593-605.

Cason, N., Astésano, C., \& Schön, D. (2015). Bridging music and speech rhythm: Rhythmic priming and audio-motor training affect speech perception. Acta Psychologica, 155, 43-50.

Cason, N., \& Schön, D. (2012). Rhythmic priming enhances the phonological processing of speech. Neuropsychologia, 50(11), 2652-2658.

Christophe, A., Millotte, S., Bernal, S., \& Lidz, J. (2008). Bootstrapping lexical and syntactic acquisition. Language and Speech, 51(1-2), 61-75.

Church, R. M., \& Lacourse, D. M. (1998). Serial pattern learning of temporal intervals. Animal Learning \& Behavior, 26(3), 272-289.

Cook, P., Rouse, A., Wilson, M., \& Reichmuth, C. (2013). A California sea lion (Zalophus californianus) can keep the beat: Motor entrainment to rhythmic auditory stimuli in a non vocal mimic. Journal of Comparative Psychology, 127(4), 412.

Emmendorfer, A. K., Correia, J. M., Jansma, B. M., Kotz, S. A., \& Bonte, M. (2020). ERP mismatch response to phonological and temporal regularities in speech. Scientific Reports, 10(1), 1-12.

Garcia, M., \& Ravignani, A. (2020). Acoustic allometry and vocal learning in mammals. Biology Letters, 16(7), 20200081.

Grahn, J. A., \& Brett, M. (2007). Rhythm and beat perception in motor areas of the brain. Journal of Cognitive Neuroscience, 19(5), 893-906.

Hannon, E. E., \& Johnson, S. P. (2005). Infants use meter to categorize rhythms and melodies: Implications for musical structure learning. Cognitive Psychology, 50(4), 354-377.

Heinrich, T., Ravignani, A., \& Hanke, F. H. (2020). Visual timing abilities of a harbour seal (Phoca vitulina) and a South African fur seal (Arctocephalus pusillus pusillus) for suband supra-second time intervals. Animal Cognition, 23(5): 851-859.

Heldstab, S. A., Isler, K., Schuppli, C., \& van Schaik, C. P. (2020). When ontogeny recapitulates phylogeny: Fixed neurodevelopmental sequence of manipulative skills among primates. Science Advances, 6(30), eabb4685.

Höhle, B. (2009). Bootstrapping mechanisms in first language acquisition. Linguistics, 47(2), $359-382$.

Honing, H. (2012). Without it no music: Beat induction as a fundamental musical trait. Annals of the New York Academy of Sciences, 1252(1), 85-91.

Hyland Bruno, J. (2017). Song rhythm development in zebra finches. City University of New York.

Jones, M. R. (2010). Attending to sound patterns and the role of entrainment. In Nobre, A. C. \& Coull, J. T. (eds.) Attention and time (pp. 317-330). Oxford University Press.

Kotz, S. A., Ravignani, A., \& Fitch, W. T. (2018). The evolution of rhythm processing. Trends in Cognitive Sciences, 22(10), 896-910.

Lattenkamp, E. Z., \& Vernes, S. C. (2018). Vocal learning: A language-relevant trait in need of a broad cross-species approach. Current Opinion in Behavioral Sciences, 21, 209-215.

Lipkind, D., Marcus, G. F., Bemis, D. K., Sasahara, K., Jacoby, N., Takahasi, M, ... Tchernichovski, O. (2013). Stepwise acquisition of vocal combinatorial capacity in songbirds and human infants. Nature, 498(7452), 104-108.

Martins, P. T., \& Boeckx, C. (2020). Vocal learning: Beyond the continuum. PLoS Biology, 18(3), e3000672.

Merker, B. H., Madison, G. S., \& Eckerdal, P. (2009). On the role and origin of isochrony in human rhythmic entrainment. Cortex, 45(1), 4-17.

Milne, A. J., \& Herff, S. A. (2020). The perceptual relevance of balance, evenness, and entropy in musical rhythms. Cognition, 203, 104233.

Miton, H., Wolf, T., Vesper, C., Knoblich, G., \& Sperber, D. (2020). Motor constraints influence cultural evolution of rhythm. Proceedings of the Royal Society B, 287 (1937), 20202001.

Ng, L., Garcia, J. E., Dyer, A. G., \& Stuart-Fox, D. (2020). The ecological significance of time sense in animals. Biological Reviews, 96(2), 526-540.

Nowicki, S., \& Searcy, W. A. (2014). The evolution of vocal learning. Current opinion in Neurobiology, 28, 48-53.

Norton, P. (2019). Isochronous rhythmic organization of learned animal vocalizations. Doctoral dissertation.

Patek, S. N., \& Caldwell, R. L. (2006). The stomatopod rumble: Low frequency sound production in Hemisquilla californiensis. Marine and Freshwater Behaviour and Physiology, 39(2), 99-111.

Patel, A. D. (2006). Musical rhythm, linguistic rhythm, and human evolution. Music Perception, 24(1), 99-104.
Patel, A., Iversen, J., Bregman, M., \& Schulz, I. (2009). Studying synchronization to a musical beat in nonhuman animals. Annals of the New York Academy of Sciences, 1169(1), 459-469.

Ravignani, A. (2017). Interdisciplinary debate: Agree on definitions of synchrony. Nature, $545,158$.

Ravignani, A., Bowling, D. L., \& Fitch, W. (2014). Chorusing, synchrony, and the evolutionary functions of rhythm. Frontiers in Psychology, 5, 1118.

Ravignani, A., Kello, C. T., De Reus, K., Kotz, S. A., Dalla Bella, S., Méndez-Aróstegui, M., ...de Boer, B. (2019). Ontogeny of vocal rhythms in harbor seal pups: An exploratory study. Current Zoology, 65(1), 107-120.

Ravignani, A., \& Madison, G. (2017). The paradox of isochrony in the evolution of human rhythm. Frontiers in Psychology, 8, 1820.

Salami, A., Wåhlin, A., Kaboodvand, N., Lundquist, A., \& Nyberg, L. (2016) Longitudinal evidence for dissociation of anterior and posterior MTL resting-state connectivity in aging: Links to perfusion and memory. Cerebral Cortex, 26(10), 3953-3963.

Schusterman, R. J. (1977). Temporal patterning in sea lion barking (Zalophus californianus). Behavioral Biology, 20(3), 404-408.

Wilson, M., \& Cook, P. F. (2016). Rhythmic entrainment: Why humans want to, fireflies can't help it, pet birds try, and sea lions have to be bribed. Psychonomic Bulletin \& Review, 23(6), 1647-1659.

Wirthlin, M., Chang, E. F., Knörnschild, M., Krubitzer, L. A., Mello, C. V., Miller, C. T., ... Yartsev, M. M. (2019). A modular approach to vocal learning: Disentangling the diversity of a complex behavioral trait. Neuron, 104(1), 87-99.

\section{A boldly comparative approach will strengthen co-evolutionary accounts of musicality's origins}

\section{Luke Rendella ${ }^{\circledR}$, Emily L. Doolittle ${ }^{b}$, Ellen C. Garland ${ }^{c}$ (i) and Alex South ${ }^{d}$}

${ }^{\mathrm{a}}$ Centre for Social Learning and Cognitive Evolution \& Sea Mammal Research Unit, University of St Andrews, St Andrews, Fife KY16 9TH, UK; ${ }^{\mathrm{b}}$ Research and Knowledge Exchange, Royal Conservatoire of Scotland, Glasgow G2 3DB, UK; ${ }^{\mathrm{C} C e n t r e ~ f o r ~ S o c i a l ~ L e a r n i n g ~ a n d ~ C o g n i t i v e ~ E v o l u t i o n ~ \& ~ S e a ~ M a m m a l ~ R e s e a r c h ~}$ Unit, University of St Andrews, St Andrews, Fife KY16 8LB, UK and ${ }^{\text {dCentre for }}$ Social Learning and Cognitive Evolution \& Sea Mammal Research Unit, University of St Andrews, and Royal Conservatoire of Scotland, Glasgow G2 3DB, UK. ler4@st-andrews.ac.uk; www.st-andrews.ac.uk/biology/people/ler4 e.doolittle@rcs.ac.uk; https://www.rcs.ac.uk/staff/emily-doolittle ecg5@st-andrews.ac.uk; www.st-andrews.ac.uk/biology/people/ecg5; a.south@rcs.ac.uk; www.alexsouth.org

\section{doi:10.1017/S0140525X20001715, e89}

\section{Abstract}

Focus on the evolutionary origins of musicality has been neglected relative to attention on language, so these new proposals are welcome stimulants. We argue for a broad comparative approach to understanding how the elements of musicality evolved, and against the use of overly simplistic evolutionary accounts.

\begin{abstract}
"there is no reason to imagine that it emerged one day wholly made by evolution ... recognize that there is no 'music in and of itself,' no musical essence, but only some distinct capacities that one day converged toward what we today call music." (Molino, 2000, p. 169)
\end{abstract}

It is exciting to see the evolution of music, or rather, musicality (Honing, 2018), neglected in the evolutionary sciences relative 